\title{
RESEARCH
}

\section{PREDICTING THE RISK OF DYSKINESIA DEVELOPMENT IN PARKINSON'S DISEASE}

Turkish Journal of Geriatrics

DOI: 10.31086/tigeri.2020.157

2020; 23(2): 224-229

- Ahmet ADIGÜZEL ${ }^{1}$ (D)

- Ünal ÖZTÜRK' ${ }^{1}$ (D)

- Sibel ALTINAYAR ${ }^{2}$

CORRESPONDANCE

Unal ÖZTÜRK

Health Science University, Gazi Yasargil

Education And Research Hospital, Neurology,

Diyarbakir, TURKEY.

Phone: +905072053407

e-mail: drunalozturk@gmail.com

Received: January 10, 2020

Accepted: April 22, 2020

${ }^{1}$ Health Science Universiity Gazi Yasargil

Education And Research Hospital, Neurology,

Diyarbakir, TURKEY

2 Inonu University, Neurology, Malatya,

TURKEY

\section{Abstract}

Background: Parkinson's Disease is a neurodegenerative disease characterized by motor and non-motor findings. Motor findings generally start with involvement of a single extremity and progress throughout life; asymmetrical involvement continues with clinical findings typically predominant in the first involved side. Parkinson's Disease is divided into two phenotypes, tremor dominant, and nontremor dominant.

We aimed to investigate and compare the incidence of dyskinesia development and other clinical parameters such as age, gender, disease duration, and treatment between the tremor dominant and non-tremor dominant phenotypes of idiopathic Parkinson's Disease.

Methods: We conducted a retrospective study of 502 patients (183 females, 319 male) with idiopathic Parkinson's Disease.

Results: Two hundred eighty-five (56\%) patients had tremor dominant phenotype and 217 (44\%) had non-tremor dominant. Dyskinesia was observed in $29 \%$ of the patients overall, $24 \%$ of the tremor dominant patients, and $35 \%$ of the non-tremor dominant patients. Dyskinesia incidence was significantly greater in the non-tremor dominant group than the tremor dominant group $(p=0.006)$. Average age at diagnosis in patients who developed dyskinesia $57 \pm 12.9$ years and who did not $64 \pm 11.1$ years. Thirty-four ( 24 tremor dominant, 10 non-tremor dominant) patients had a family member with Parkinson's Disease. Thirteen patients ( 5 tremor dominant, 8 non-tremor dominant) with a positive family history developed dyskinesia. The incidence of dyskinesia development according to clinical phenotype was independent from family history and was greater in the NTD group $(p=0.02)$. Levodopa treatment was received by $421(83 \%)$ patients; the incidence of dyskinesia development who used levodopa was 33\% ( $p<0.001)$.

Conclusions: Patients with non-tremor dominant phenotype of Idiopathic Parkinson's Disease have a significantly higher risk of developing dyskinesia and should be closely clinically followed.

Keywords: Parkinson disease; Dyskinesias; Neurodegenerative diseases. 


\section{INTRODUCTION}

Idiopathic Parkinson's Disease (PD) is the most frequently observed neurodegenerative disease after Alzheimer's disease, affecting approximately $1 \%$ of people over the age of 65 and $5 \%$ of people over the age of 85 (1). Significant motor findings in PD include resting tremor, bradykinesia, rigidity, and postural instability. These are generally progressive and asymmetric with predominance in the first involved side (2). In 1990, the Parkinson's Study Group divided PD patients into two clinical phenotypes, tremor dominant (TD) and non-tremor dominant (NTD) (3). Non-tremor dominant (akinetic-rigid type) PD proceeds more progressively than TD and develops more functional disability $(4,5)$.

Tremor in PD emerges typically during rest and is observed in $60-75 \%$ of patients (2). The tremor characteristically involves the thumb and is referred to as a "money counting tremor" or "pill rolling tremor." In patients with NTD, rigidity, walking disorder, stiffening, and postural instability are the primary symptoms. Bradykinesia is a characteristic clinical finding in both phenotypes and manifests as generally slow movement and impaired fine motor movements that require skill.

Dyskinesia is a common motor complication of treatment and presents as hyperkinetic involuntary movements that are frequently choreiform in nature. The most important dyskinesia risk factors are levodopa (LD) dose and treatment duration. Treatment-related dyskinesias are classified based on their temporal relationship with medication level (e.g., peak dose, end dose). Although dyskinesia in PD has been well-described, there is no clear consensus on its pathophysiologic mechanism. Therefore, in this study, we evaluated demographic and clinical features between the TD and NTD phenotypes of PD and their effect on dyskinesia development.

\section{METHODS}

Study approval was obtained from the ethics committee of our hospital (378 no and 29/11/2019 date) and principles of the Declaration of Helsinki Declaration were followed. All study patients provided written informed consent.

We retrospectively identified patients diagnosed with idiopathic PD using hospital medical records for inclusion in this study. Data regarding patient demographics and clinical characteristics, clinical stage of PD as defined by the Modified Hoehn and Yahr (H\&Y) Staging Scale, clinical phenotype of PD (TD and NTD), treatment, and dyskinesia incidence were recorded. Patients with mixed (undefined dominant) phenotype were not included for analysis.

Statistical analysis was conducted using SPSS for Windows, version 20.0 software (IBM Corp., Armonk, NY, USA). The chi-square test was used for comparison of categorical variables. Normal distribution of continuous data was determined using the Kolmogorov-Smirnov test. For comparison of continuous variables between groups, the Student's t-test was used for normally distributed data and the MannWhitney $U$ test for non-normally distributed data. For correlation analysis, the Spearman test was used for non-parametric data and the Pearson test for parametric data. $P<0.05$ was considered statistically significant.

\section{RESULTS}

Five hundred two patients, including 183 (36\%) women and 319 (64\%) men were included in this study. Detailed clinical data are shown in Table 1. Dyskinesia was observed in 144 (29\%) patients (55 women, 89 men). Average age at PD diagnosis was $62.3 \pm 11.97$ years overall. Average age at diagnosis was younger in patients who developed dyskinesia than those who did not ( $57 \pm 12.9$ years vs. $64 \pm 11.1$ years). Two hundred eighty-five (56\%) patients had TD clinical phenotype and 217 (44\%) had NTD phenotype. Of the 285 TD patients, 68 (24\%) developed dyskinesia and $35 \%$ (76) did not. Seventy-six (35\%) of the 217 NTD patients 
Table 1. Clinical Properties and Phenotype Relationship.

\begin{tabular}{|c|c|c|c|c|c|c|c|c|c|c|c|c|c|c|}
\hline & 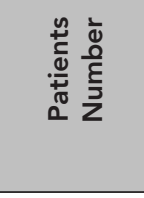 & 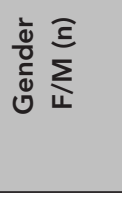 & 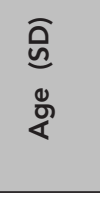 & 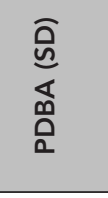 & 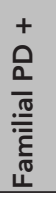 & 口 & $\overleftarrow{\Delta}$ & $\begin{array}{l}\frac{c}{\leq} \\
\infty \\
0 \\
\frac{1}{\Sigma}\end{array}$ & 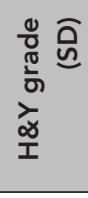 & 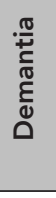 & $\begin{array}{l}\frac{4}{0} \\
i \\
\vdots \\
0\end{array}$ & 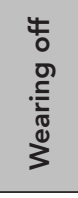 & 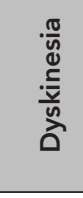 & ณ \\
\hline 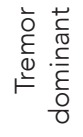 & $285(\% 56)$ & 113/172 & $\begin{array}{r}68,35 \\
(10,62)\end{array}$ & $\begin{array}{r}62,20 \\
(11,85)\end{array}$ & 24 & 219 & 187 & 177 & $\begin{array}{r}2,27 \\
(0,92)\end{array}$ & 87 & 40 & 35 & 68 & 33 \\
\hline 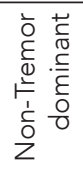 & $217(\% 44)$ & $70 / 147$ & $\begin{array}{r}68,19 \\
(11,26)\end{array}$ & $\begin{array}{r}62,46 \\
(12,13)\end{array}$ & 10 & 202 & 141 & 132 & $\begin{array}{r}2,57 \\
(0,93)\end{array}$ & 84 & $49^{*}$ & $46^{\star *}$ & $76^{* * *}$ & 29 \\
\hline
\end{tabular}

* $\mathrm{p}=0,046 \quad$ ** $\mathrm{p}=0,024 \quad$ *** $\mathrm{p}=0,006$

PDBA: Parkinson's Disease Beginning Age F: Female M:Male PD: Parkinson's Disease LD: Levodopa DA: Dopamine Agonist MAO-B inh: Monoamine Oxidase B inhibitor(rasagiline) DDS: Dopamine Dysregulation Syndrome

developed dyskinesia, whereas 141 (65\%) (141) did not. The incidence of dyskinesia development was significantly higher in the NTD group than the TD group ( $p=0.006$ ) (Table 2 ).

Most study participants (217) were H\&Y stage 2. Thirty-four (17\%) patients had a family member with PD; of these, 24 (70\%) had TD clinical phenotype and 10 (30\%) had NTD. Thirteen (38\%) patients with a positive family history developed dyskinesia (5 TD patients, 8 NTD patients). The incidence of dyskinesia development according to clinical phenotype was independent from family history and was greater in the NTD group
( $p=0.02)$. LD treatment was received by 421 (83\%) patients; the incidence of dyskinesia development was significantly greater in these patients compared to those who did not receive LD (33\% vs. $2.5 \% ; p<0.001)$. We detected no significant differences in dyskinesia incidence between the other treatments (dopamine agonists (DA), and monoamine oxidase type B inhibitors).

\section{DISCUSSION}

Pathophysiological mechanisms

PD is a movement disorder characterized by

Table 2. Parameters Affecting Dyskynesia.

\begin{tabular}{|c|c|c|c|c|c|c|c|c|}
\hline & \multirow{2}{*}{$\begin{array}{r}\text { Patients } \\
\text { number } \\
(\mathrm{F} / \mathrm{M})\end{array}$} & \multirow{2}{*}{$\begin{array}{r}\text { PDBA. } \\
\text { (SD) }\end{array}$} & \multirow[b]{2}{*}{ TD } & \multirow[b]{2}{*}{ NTD } & \multicolumn{2}{|c|}{ Use of LD } & \multicolumn{2}{|c|}{ Familial PD + } \\
\hline & & & & & yes & no & $\begin{array}{r}\text { Yes(TD/ } \\
\text { NTD) }\end{array}$ & $\begin{array}{r}\text { No(TD/ } \\
\text { NTD) }\end{array}$ \\
\hline \multirow{2}{*}{ Dyskinesia + } & $144(55 / 89)$ & 57,98 & 68 & $76^{*}$ & $142^{\star \star}$ & 2 & $13(5 / 8)$ & $76(36 / 40)^{\star \star \star \star}$ \\
\hline & (\%28) & $(12,93)$ & $(\% 24)$ & (\%35) & (\%34) & (\%3) & (\%38) & (\%44) \\
\hline \multirow{2}{*}{ Dyskinesia - } & $358(128 / 230)$ & 64,06 & 217 & 141 & 279 & 76 & $21(19 / 2)$ & $95(52 / 43)$ \\
\hline & $(\% 72)$ & $(11,10)$ & $(\% 76)$ & (\%35) & (\%66) & (\%97) & (\%62) & (\%56) \\
\hline \multirow{2}{*}{ Total } & $502(183 / 319)$ & 62,31 & 285 & 217 & 421 & 78 & 34 & 171 \\
\hline & (\%100) & $(11,96)$ & $(\% 100)$ & $(\% 100)$ & (\%100) & (\%100) & $\% 100$ & (\%100) \\
\hline
\end{tabular}

${ }^{*} p=0,006{ }^{* *} p=0,000 * * * p=0,02$.

PDBA: Parkinson Disease Beginning Age TD: Tremor Dominant NTD: Non-tremor Dominant LD: Levodopa. 
involvement of the basal ganglia and other structures of the extrapyramidal system (e.g., cerebellum). Histopathologically, dopaminergic neuron loss, and Lewy particles, which are eosinophilic intracytoplasmic protein inclusions, are observed in the substantia nigra pars compacta (SNc) (6). Although both TD and NTD phenotypes of PD exhibit these pathologic findings, previous studies have shown many histopathologic and pathophysiologic differences between the two. In an autopsy study that included 27 NTD and 18 TD cases, neuron loss in the SNc and locus coeruleus was less in TD cases (7). A similar 2012 study also showed less locus coeruleus involvement in TD cases (8). Differences between TD and NTD phenotypes have been shown in living patients as well. A functional magnetic resonance imaging study of 17 PD patients (9TD, 8 NTD) and 14 control patients that evaluated striatothalamocortical and cerebellothalamocortical circulation showed greater circulation in both anatomic territories in TD patients than the other groups (9). The presynaptic dopaminergic system can be evaluated by calculating the amount of striatal dopamine transporters using single-photon emission computed tomography (SPECT) scanning. In a previous SPECT study, a significant relationship was found between striatal iodine-123 (123I) fluoropropyl (FP) carbomethoxy-3 $\beta$-(4iodophenyltropane) (CIT) uptake and the severity of disease in akinetic-rigid patients (NTD phenotype), but not in patients with tremor (TD phenotype) (10). The above findings suggest that the two PD phenotypes have different underlying pathologic mechanisms.

To date, there is no clear consensus regarding the pathophysiologic mechanisms of dyskinesia development. One theory based on dopaminedepleted animal models and human PD studies postulates that dyskinesia develops as a result of dysfunction of the glutaminergic transmission from the cortex to striatal medium spiny neurons (MSNs) (11). MSNs are characterized by thorn-like structures projecting to dendrites that receive excitatory glutaminergic impulses from the cerebral cortex. These projections are in contact with post-synaptic D1/D2 receptors. In the presynaptic area, the decreased dopamine and increased glutamate resulting from PD provide a basis for dyskinesia development as a result of increased sensitivity to dopamine (12).

\section{Clinical features}

Tremor is observed in approximately $2 / 3$ of PD patients (4), and the progression of PD is slower and functional loss is less in this subgroup (5). In our study, $56 \%$ of the patients had TD phenotype. Dyskinesia can develop in both TD and NTD phenotypes; we found dyskinesia incidence rates of $24 \%$ in TD patients, 35\% in NTD patients, and $28 \%$ in all patients. This study additionally showed that dyskinesia incidence is higher in patients with NTD phenotype $(p=0.006)$, similar to a previous 2013 study (13), and in agreement with a 2014 123I-FP-CIT SPECT study that determined that dyskinesia risk is greater in NTD patients and suggested that TD and NTD patients require different therapeutic approaches to dyskinesia (14).

We also considered other parameters that may affect the development of dyskinesia such as gender, duration of diagnosis, patient age at diagnosis, and treatment. Patients with dyskinesia in our study were diagnosed at an earlier age: the average age of those who developed dyskinesia was 57.9 years, compared to 64 years in those without dyskinesia. This suggests that individuals diagnosed with PD at an early age are more prone to dyskinesia development. In the aforementioned 2013 study, dyskinesia developed in 58\% of the patients aged $25-40$ years and $23.5 \%$ of the patients aged $\geq 40$ years old, further supporting the notion that young patients are at higher risk for developing dyskinesia (13). We additionally hypothesize that dysfunction of glutamate transmission, which is considered the first step in dyskinesia development, started at the moment of diagnosis or even before in individuals who were diagnosed at an early age. In other words, the younger age of individuals with dyskinesia leads 
us to believe that a mechanism unrelated to LD, which is the main agent triggering dyskinesia, previously set the stage.

LD generally results in significant recovery of PD symptoms in the first years after treatment initiation. However, after 5 years, side effects such as motor complications (dyskinesias, on-off and wearing off symptoms), autonomic disorders, and psychosis are commonly observed. Until recently, there was consensus about starting early treatment with DA or MAO-B inhibitors and delaying the transition to LD as late as possible to decrease the risk of dyskinesia. However, in a 2018 compilation, LD was emphasized as the most effective agent for motor symptoms and early transition to LD was suggested; this compilation also emphasized that dyskinesia may be related to the dose and posology of the medication rather than treatment duration (15). In our study, we examined the relationship between treatments and dyskinesia and detected that LD has a dominant effect on rasagiline (MAO-B inhibitor) and DA for dyskinesia. One hundred forty-two patients had dyskinesia among the 421 patients receiving LD and only 2 patients had dyskinesia among the 81 patients not using LD $(p<0.001)$. We also obtained the same result when we evaluated the relationship between diagnosis duration $(\leq 5$ years vs. $>5$ years), LD and dyskinesia. In other

\section{REFERENCES}

1. Martinez-Martin P, Rodriguez-Blazquez C, Kurtis MM, Chaudhuri KR, NMSS Validation Group. The impact of non-motor symptoms on health-related quality of life of patients with Parkinson's disease. Movement Disorders 2011 ;26(3):399-406. (PMID: 21264941)

2. Fernandez HH. 2015 Update on Parkinson disease. Cleve Clin J Med. 2015 ;82(9):563-8. (PMID:26366951).

3. Jankovic J, McDermott M, Carter J, et al. Variable expression of Parkinson's disease: A baseline analysis of the DATATOP cohort. Neurology $1990 ; 40(10)$ :152934. (PMID:2215943).

4. Kalia LV, Lang AE. Parkinson disease in 2015: evolving basic, pathological and clinical concepts in PD. Nature words, LD is a strong risk factor for dyskinesia independent from duration of disease. In a 2014 multicenter study, LD dose reduction resulted in decreased severity and frequency of dyskinesia (16). This result suggests that increased sensitivity to dopamine in D1 receptors keep continue in long term period thanks to LD or glutamate collection keeps induced dyskinesia to continue alone.

In conclusion, we examined numerous clinical parameters as potential risk factors for dyskinesia development in PD. Patients with NTD phenotype appear to have a significant and independent risk and should be closely clinically followed.

\section{Acknowledgement}

\section{Statement of Ethics}

The study was approved by the Ethics Committee of University of Health Sciences, Diyarbakır Gazi Yasargil Education and Research Hospital. The study complied with the Declaration of Helsinki.

\section{Disclosure Statement}

The authors have no conflicts of interest to declare.

\section{Funding Sources}

This research did not receive any specific grant from funding agencies in the public, commercial, or not-for-profit sectors. reviews Neurology 2016 ;12(2):65. (PMID:26782330).

5. Marras C, Lang A. Parkinson's disease subtypes: lost in translation? J Neurol Neurosurg Psychiatry 2013 ;84(4):409-15. (PMID: 22952329).

6. Goedert M, Spillantini MG, Del Tredici K, Braak H. 100 years of Lewy pathology. Nature Reviews Neurology $2013 ; 9(1): 13$. (PMID: 23183883).

7. Paulus $W$, Jellinger $K$. The neuropathologic basis of different clinical subgroups of Parkinson's disease. Journal of Neuropathology \& Experimental Neurology $1991 ; 50(6): 743-55$. (PMID:1748881).

8. Van de Berg WD, Hepp DH, Dijkstra AA, Rozemuller JA, Berendse HW, Foncke E. Patterns of alpha- 
synuclein pathology in incidental cases and clinical subtypes of Parkinson's disease. Parkinsonism \& related disorders $2012 ; 18: S 28-S 30$. (PMID:22166446).

9. Lewis MM, Du G, Sen S, et al. Differential involvement of striato-and cerebello-thalamo-cortical pathways in tremor-and akinetic/rigid-predominant Parkinson's disease. Neuroscience $2011 ; 177: 230-9$. (PMID:21211551).

10. Eggers C, Kahraman D, Fink GR, Schmidt M, Timmermann L. Akinetic-rigid and tremor-dominant Parkinson's disease patients show different patterns of FP-CIT Single photon emission computed tomography. Movement Disorders 2011 ;26(3):41623. (PMID:21264942).

11. Picconi B, Hernández LF, Obeso JA, Calabresi P. Motor complications in Parkinson's disease: Striatal molecular and electrophysiological mechanisms of dyskinesias. Movement Disorders 2018 ;33(6):867-76. (PMID:29219207).

12. Gerfen CR, Surmeier DJ. Modulation of striatal projection systems by dopamine. Annual review of neuroscience $2011 ; 34: 441-66$. (PMID: 21469956).
13. Zhang Y-h, Tang B-s, Song C-y, et al. The relationship between the phenotype of Parkinson's disease and levodopa-induced dyskinesia. Neuroscience letters 2013 ;556:109-12. (PMID: 24135335).

14. Kaasinen V, Kinos M, Joutsa J, Sappanen M, Noponen T. Differences in striatal dopamine transporter density between tremor dominant and non-tremor Parkinson's disease. European journal of nuclear medicine and molecular imaging $2014 ; 41(10): 1931-7$. (PMID: 24867256)

15. Reich SG, Savitt JM. Parkinson's Disease. The Medical clinics of North America 2019;103(2):337-50. (PMID: 30704685).

16. PD Med Collobretive Group, Gray R, Ives N, et al. Long-term effectiveness of dopamine agonists and monoamine oxidase B inhibitors compared with levodopa as initial treatment for Parkinson's disease (PD MED): a large, open-label, pragmatic randomised trial. The Lancet 2014; 384(9949):1196-205. (PMID: 24928805). 